\title{
On the rigidity of molecular graphs
}

\author{
Bill Jackson*and Tibor Jordán ${ }^{\dagger}$
}

November 16, 2007

\begin{abstract}
The rigidity of squares of graphs in three-space has important applications to the study of flexibility in molecules. The Molecular Conjecture, posed in 1984 by T-S. Tay and W. Whiteley, states that the square $G^{2}$ of a graph $G$ of minimum degree at least two is rigid if and only if $G$ has six spanning trees which cover each edge of $G$ at most five times. We give a lower bound on the degrees of freedom of $G^{2}$ in terms of forest covers of $G$. This provides a self-contained proof that the existence of the above six spanning trees is a necessary condition for the rigidity of $G^{2}$. In addition, we prove that the truth of the Molecular Conjecture would imply that our lower bound is tight, and would also imply that a conjecture of Jacobs on 'independent' squares is valid.
\end{abstract}

\section{Introduction}

One of the main unsolved problems in combinatorial rigidity is the characterization of rigid graphs in three-space. There exist some partial results, see for example $[4,5,6]$, but the general problem appears to be difficult, see $[3,18]$. In this paper we consider an important special case, which has been a focus of recent research: characterize when the square of a graph is rigid, where the square $G^{2}$ of a graph $G$ is obtained from $G$ by adding a new edge $u v$ for each pair $u, v \in V(G)$ of distance two in $G$, see Figure 1.

${ }^{*}$ School of Mathematical Sciences, Queen Mary, University of London, Mile End Road, London E1 4NS, England. e-mail: b.jackson@qmul.ac.uk. This work was supported by an International Joint Project grant from the Royal Society.

${ }^{\dagger}$ Department of Operations Research, Eötvös University, Pázmány Péter sétány 1/C, 1117 Budapest, Hungary. e-mail: jordan@cs.elte.hu. Supported by the MTA-ELTE Egerváry Research Group on Combinatorial Optimization and the Hungarian Scientific Research Fund grant no. T049671, T60802. 

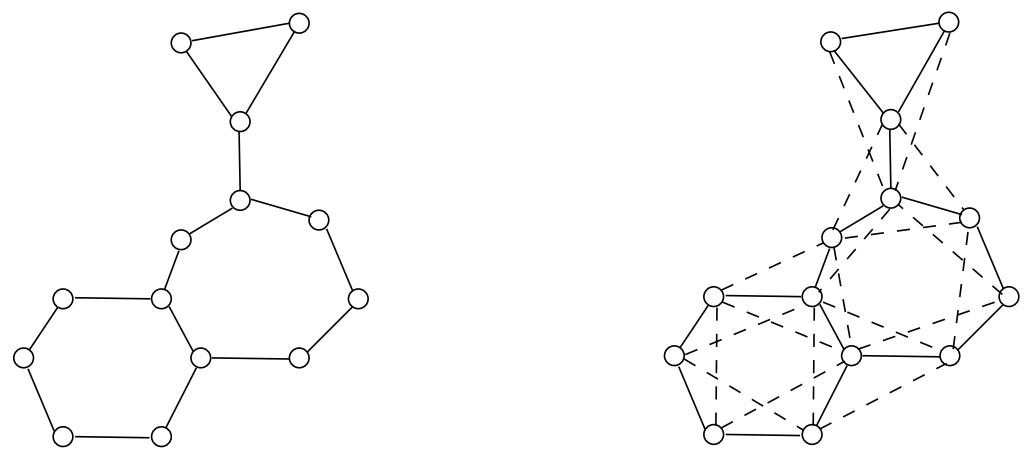

Figure 1: A graph $G$ and its square $G^{2}$.

Squares of graphs are sometimes called molecular graphs, because they are used to study the flexibility of molecules, particularly biomolecules such as proteins $[19,22]$. Franzblau [1, 2] has developed combinatorial algorithms for computing lower and upper bounds on the degrees of freedom of molecular graphs using ear-decompositions of graphs.

The Molecular Conjecture, due to Tay and Whiteley [15, Conjecture 1], states that the square $G^{2}$ of a graph $G$ of minimum degree at least two is rigid if and only if $G$ has six spanning trees which cover each edge of $G$ at most five times. The conjecture indicates that the problem of determining when molecular graphs are rigid may be significantly easier than the problem for arbitrary graphs. Our main result (Theorem 4.1) gives a lower bound on the degrees of freedom of $G^{2}$ using forest covers of $G$. This provides a self-contained proof that the existence of six spanning trees which cover each edge of $G$ at most five times is a necessary condition for the rigidity of $G^{2}$. We show that the truth of the conjecture would imply that our lower bound on the degrees of freedom of $G^{2}$ is tight. We also show that it would imply a conjecture of Jacobs [12] that a molecular graph has no 'redundant edges' if and only if it satisfies the 'Laman condition'. Related results are discussed in the concluding section.

\section{Rigid graphs and frameworks}

In this paper we consider finite graphs without loops. We will reserve the term graph for graphs without multiple edges and refer to graphs which 
may contain multiple edges as multigraphs. The multigraph obtained from a graph $G=(V, E)$ by replacing each edge $e \in E$ by $k$ copies of $e$ is denoted by $k G$.

A (three-dimensional bar-and-joint) framework $(G, p)$ is a graph $G=$ $(V, E)$ and a map $p: V \rightarrow \mathbb{R}^{3}$. We consider the framework to be a straight line realization of $G$ in $\mathbb{R}^{3}$. The rigidity matrix of the framework is a matrix $R(G, p)$ of size $|E| \times 3|V|$. For each edge $v_{i} v_{j} \in E$, the entries in the row corresponding to the edge $v_{i} v_{j}$ are defined as follows: the three columns corresponding to the vertex $v_{i}$ contain the three coordinates of $p\left(v_{i}\right)-p\left(v_{j}\right)$; the three columns corresponding to the vertex $v_{j}$ contain the three coordinates of $p\left(v_{j}\right)-p\left(v_{i}\right)$; the remaining entries are all zeros. See [18] for more details.

We refer to the vectors in the null space of $R(G, p)$ as infinitesimal motions of $(G, p)$. This definition is motivated by the fact that each continuous motion of $(G, p)$ which preserves all 'edge lengths' gives rise to an infinitesimal motion, see [18]. The null space of $R(G, p)$ will have dimension at least six since the translations and rotations of $\mathbb{R}^{3}$ give rise to six linearly independent infinitesimal motions (when $|V| \geq 3$ ). Thus,

$$
\operatorname{rank} R(G, p) \leq 3|V|-6 \text { when }|V| \geq 3 \text {. }
$$

We say $(G, p)$ is infinitesimally rigid if the rank of $R(G, p)$ is equal to $3|V|-6$.

A framework $(G, p)$ is generic if the ranks of its rigidity matrix, and all its submatrices, are maximum among all realizations of $G$. It is known that almost all realizations of $G$ are generic, and that each infinitesimal motion of a generic framework is 'induced' by a continuous motion of the framework. We denote the rank of the rigidity matrix of a generic realization of $G$ by $r(G)$ and refer to it as the rank of $G$. By the preceding paragraph $r(G) \leq 3|V|-6$, whenever $|V| \geq 3$. The graph $G$ is said to be rigid if either $G$ is a complete graph on at most two vertices, or $|V| \geq 3$ and $r(G)=3|V|-6$. More generally $3|V|-r(G)$, i.e. the dimension of the null space of $R(G, p)$ for a generic realization $(G, p)$ of $G$, is referred to as the degrees of freedom of $G$, and measures the flexibility of generic realizations of the graph.

Conjecture 2.1 below is the bar-and-joint version of the Molecular Conjecture. This conjecture was given by Tay and Whiteley in [15], and subsequently appeared in several different forms, see [18, 19, 20, 21, 22]. It is usually formulated in terms of 'body-and-hinge' frameworks, which will be described in Subsection 2.1. We heard of the bar-and-joint version of the conjecture in a private communication from Whiteley. We have not been able to find it explicitly in the literature. 
Conjecture 2.1 (Molecular Conjecture) Let $G$ be a graph with minimum degree at least two. Then $G^{2}$ is rigid if and only if $5 G$ contains six edge-disjoint spanning trees.

We may extend Conjecture 2.1 to a conjecture which characterizes the degrees of freedom of a molecular graph. Let $G=(V, E)$ be a graph. For a family $\mathcal{F}$ of pairwise disjoint subsets of $V$ let $E_{G}(\mathcal{F})$ denote the set, and $e_{G}(\mathcal{F})$ the number, of edges of $G$ connecting distinct members of $\mathcal{F}$. For a partition $\mathcal{P}$ of $V$ let

$$
\operatorname{def}_{G}(\mathcal{P})=6(|\mathcal{P}|-1)-5 e_{G}(\mathcal{P})
$$

denote the deficiency of $\mathcal{P}$ in $G$ and let

$$
\operatorname{def}(G)=\max \left\{\operatorname{def}_{G}(\mathcal{P}): \mathcal{P} \text { is a partition of } V\right\} .
$$

We say that a partition $\mathcal{P}$ of $V$ is a tight partition of $G$ if $\operatorname{def}_{G}(\mathcal{P})=\operatorname{def}(G)$. Note that $\operatorname{def}(G) \geq 0$ since $\operatorname{def}_{G}(\{V\})=0$. Theorem 2.3 below implies that $\operatorname{def}(G)$ is equal to the minimum number of edges which have to be added to $5 G$ in order to create six edge-disjoint spanning trees. The following conjecture asserts that there is a close relationship between $\operatorname{def}(G)$ and $r\left(G^{2}\right)$.

Conjecture 2.2 Let $G=(V, E)$ be a graph with minimum degree at least two. Then

$$
r\left(G^{2}\right)=3|V|-6-\operatorname{def}(G) .
$$

We may deduce that Conjecture 2.2 implies Conjecture 2.1 by using a result, proved independently by Nash-Williams and Tutte.

Theorem 2.3 [13, 16] Let $H=(V, E)$ be a multigraph and let $k$ be a positive integer. Then $H$ contains $k$ edge-disjoint spanning trees if and only if

$$
e_{H}(\mathcal{P}) \geq k(|\mathcal{P}|-1)
$$

for all partitions $\mathcal{P}$ of $V$. 


\subsection{Body-and-hinge frameworks}

We give a brief informal description of the molecular conjecture for bodyand-hinge frameworks in this subsection. It is independent from the rest of the paper, where we will only be concerned with bar-and-joint frameworks. In a (three-dimensional) body-and-hinge framework, vertices are represented by rigid bodies and each edge by a hinge which connects the corresponding pair of bodies. The bodies are free to move continuously in $\mathbb{R}^{3}$ subject to the constraint that the relative motion of any two bodies joined by a hinge is a rotation about the hinge. The framework is rigid if every such motion preserves the distances between all pairs of points belonging to different rigid bodies, i.e. the motion extends to an isometry of $\mathbb{R}^{3}$. As in bar-and-joint frameworks, the infinitesimal motions of a body-and-hinge framework can be defined as the vectors in the null space of an associated rigidity matrix, and the framework is infinitesimally rigid if the null space of this matrix has dimension six, see [11]. Tay [14] and Whiteley [17] independently showed that a graph $G$ has an infinitesimally rigid body-and-hinge realization if and only if $5 G$ has six edge-disjoint spanning trees.

A molecular framework is a body-and-hinge framework in which the lines containing the hinges incident to each body are constrained to meet at a common point. The study of these frameworks is motivated by their application in molecular biology. In this application we model a molecule by a body-and-hinge framework which represents each atom as a body. The relative motion of two atoms which are linked by a bond is constrained to be a rotation about the line through the centre of the atoms. This gives rise to a molecular framework whose rigidity properties provide information about the flexibility of the molecule. Tay and Whiteley conjectured in [15, Conjecture 1] that if $G$ has an infinitesimally rigid body-and-hinge realization then $G$ has an infinitesimally rigid realization as a molecular framework. The truth of this conjecture would imply that the results on generic bodyand-hinge frameworks (e.g. the characterization of rigidity, algorithms for computing the degrees of freedom, etc.) extend to molecular frameworks.

Intuitively, the connection between the rigidity of a bar-and-joint realization $\left(G^{2}, p\right)$ of $G^{2}$ and the rigidity of a molecular realization of $G$ can be seen by observing that each vertex $v$ and its neighbours in $G$ induce a complete subgraph in $G^{2}$ which acts as a rigid body in $\left(G^{2}, p\right)$. The relative motion of two such bodies corresponding to adjacent vertices $u, v$ of $G$ is constrained to be a rotation about the line containing the points $p(u)$ and $p(v)$. Whiteley [21] has formalized this connection by showing that a graph 
$G$ of minimum degree at least two has an infinitesimally rigid molecular realization if and only if $G^{2}$ has an infinitesimally rigid realization as a barand-joint framework. It follows that the above mentioned conjecture of Tay and Whiteley is equivalent to Conjecture 2.1 in this paper. See [11, 18, 22] for formal definitions and more details on body-and-hinge frameworks.

\section{Covers and partitions}

Let $G=(V, E)$ be a multigraph. For $X \subseteq V$, let $E_{G}(X)$ denote the set, and $i_{G}(X)$ the number, of edges in $G[X]$, that is, in the subgraph induced by $X$ in $G$. For $X \subset V$ let $d_{G}(X)=e_{G}(X, V-X)$ denote the degree of $X$. If $X=\{v\}$ for some $v \in V$ then we simply write $d_{G}(v)$ for the degree of $v$. The set of neighbours of $X$ (i.e. the set of those vertices $v \in V-X$ for which there exists an edge $u v \in E$ with $u \in X)$ is denoted by $N_{G}(X)$. We use $E(X), i(X), d(X)$, or $N(X)$ when the multigraph $G$ is clear from the context. A graph $G=(V, E)$ is $M$-independent if the rows of $R(G, p)$ are linearly independent in some (and hence in all) generic realizations $(G, p)$ of $G$.

A cover of $G=(V, E)$ is a collection $\mathcal{X}$ of subsets of $V$, each of size at least two, such that $\bigcup_{X \in \mathcal{X}} E(X)=E$. A cover $\mathcal{X}=\left\{X_{1}, X_{2}, \ldots, X_{m}\right\}$ of $G$ is 2 -thin if $\left|X_{i} \cap X_{j}\right| \leq 2$ for all $1 \leq i<j \leq m$. For $X_{i} \in \mathcal{X}$ let $f\left(X_{i}\right)=1$ if $\left|X_{i}\right|=2$ and $f\left(X_{i}\right)=3\left|X_{i}\right|-6$ if $\left|X_{i}\right| \geq 3$. Let $H(\mathcal{X})$ be the set of all pairs of vertices $u v$ such that $X_{i} \cap X_{j}=\{u, v\}$ for some $1 \leq i<j \leq m$. For each $u v \in H(\mathcal{X})$ let $h(u v)$ be the number of sets $X_{i}$ in $\mathcal{X}$ such that $\{u, v\} \subseteq X_{i}$ and put

$$
\operatorname{val}(\mathcal{X})=\sum_{X \in \mathcal{X}} f(X)-\sum_{u v \in H(\mathcal{X})}(h(u v)-1) .
$$

We say that a 2-thin cover $\mathcal{X}$ of a graph $G=(V, E)$ is independent if the graph $(V, H(\mathcal{X}))$ is $M$-independent. The following lemma shows that independent covers of $G$ can be used to give an upper bound on $r(G)$.

Lemma 3.1 [5, Lemma 3.2] Let $G=(V, E)$ be a graph, and let $\mathcal{X}$ be an independent 2-thin cover of $G$. Then $r(G) \leq \operatorname{val}(\mathcal{X})$.

We say that the graph $G$ is strong if $5 G$ has six edge-disjoint spanning trees, or equivalently, if $\operatorname{def}(G)=0$. A subgraph $H$ is a brick of $G$ if $H$ is a maximal strong subgraph of $G$. Thus bricks are induced subgraphs of $G$. We showed in [10] that the vertex sets of the bricks of $G$ partition $V$. We 

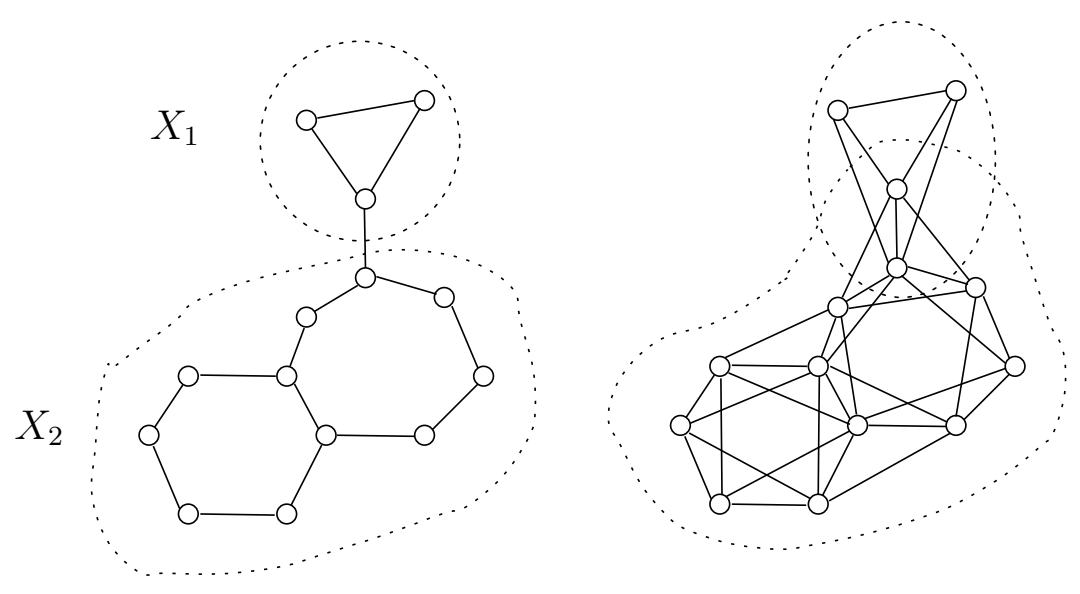

Figure 2: A graph $G=(V, E)$ and its brick partition $\left\{X_{1}, X_{2}\right\}$, together with the corresponding independent 2-thin cover $\mathcal{X}$ of $G^{2}$. We have $\operatorname{def}(G)=1$ and $\operatorname{val}(\mathcal{X})=35=3|V|-6-\operatorname{def}(G)$.

shall refer to this partition of $V$ as the brick partition of $G$, see Figure 2. We will see in Theorem 3.4 below that every tight partition of $G$ gives rise to an independent 2-thin cover $\mathcal{X}$ of $G^{2}$ with $\operatorname{val}(\mathcal{X})=3|V|-6-\operatorname{def}(G)$. This is illustrated for the brick partition in Figure 2. We show in [8] that the brick partition of $G$ can be constructed efficiently, and also that the Molecular Conjecture implies that the bricks of $G$ give rise, in a natural way, to the maximal rigid subgraphs of $G^{2}$.

We will need the following result from [10].

Lemma 3.2 [10] Let $G=(V, E)$ be a graph and $\mathcal{P}$ be a tight partition of $V$.

(a) If $\mathcal{Q} \subseteq \mathcal{P}$ with $|\mathcal{Q}| \geq 2$ and $H$ is the subgraph of $G$ induced by the union of the members of $\mathcal{Q}$, then $\operatorname{def}_{H}(\mathcal{Q}) \geq 0$.

(b) If $P \in \mathcal{P}$ and $K=G[P]$, then $K$ is strong.

(c) If $\mathcal{P}$ is a tight partition of $G$ with as few members as possible, then $\mathcal{P}$ is the brick partition of $G$.

The following lemma is a well-known result in graph rigidity theory. The operation described in the lemma will be referred to as a 0 -extension. (It is also called vertex-addition in the literature.) 
Lemma 3.3 [18, Lemma 9.1.3] Let $H=(V, E)$ be a graph and $v_{1}, v_{2}, \ldots v_{s}$ be distinct vertices of $G$ for some $s \in\{1,2,3\}$. Let $G$ be obtained from $H$ by adding a new vertex $v$ and all edges $v v_{i}$ for $1 \leq i \leq s$. Then $G$ is $M$-independent if and only if $H$ is $M$-independent.

We can now deduce the above mentioned correspondence between tight partitions and 2-thin covers.

Theorem 3.4 Let $G=(V, E)$ be a graph of minimum degree at least two and let $\mathcal{P}=\left\{P_{1}, P_{2}, \ldots, P_{t}\right\}$ be a tight partition of $V$. Let $X_{i}=P_{i} \cup N_{G}\left(P_{i}\right)$ for $1 \leq i \leq t$ and let $\mathcal{X}=\left\{X_{1}, \ldots, X_{t}\right\}$. Then $\mathcal{X}$ is an independent 2-thin cover of $G^{2}$ with $\operatorname{val}(\mathcal{X})=3|V|-6-\operatorname{def}(G)$.

Proof: We say that $\mathcal{P}$ induces a cycle of length $k$ in $G$ if there exist distinct classes $P_{1}, P_{2}, \ldots, P_{k} \in \mathcal{P}$ and distinct edges $e_{1}, e_{2}, \ldots, e_{k} \in E$ such that $e_{i} \in E_{G}\left(P_{i}, P_{i+1}\right)$ for $1 \leq i \leq k-1$ and $e_{k} \in E_{G}\left(P_{k}, P_{1}\right)$. It follows from Lemma 3.2(a) that $\mathcal{P}$ does not induce a cycle of length less than six in $G$. In particular, $e_{G}\left(\left\{P_{i}, P_{j}\right\}\right) \leq 1$ for all $1 \leq i<j \leq t$.

It is easy to see that $\mathcal{X}$ is a cover of $G^{2}$. Furthermore, since $e_{G}\left(\left\{P_{i}, P_{j}\right\}\right) \leq$ 1 for $1 \leq i<j \leq t$, and $G$ has minimum degree at least two, we have $\left|X_{i}\right|=\left|P_{i}\right|+d_{G}\left(P_{i}\right)$ and $\left|X_{i}\right| \geq 3$ for $1 \leq i \leq t$. By using the fact that $\mathcal{P}$ does not induce cycles of length two, three or four in $G$, it is also easy to verify that $\mathcal{X}$ is a 2 -thin cover with $H(\mathcal{X})=\left\{u v: u v \in E_{G}(\mathcal{P})\right\}$ and $h(u v)=2$ for all $u v \in H(\mathcal{X})$.

We claim that $K=(V, H(\mathcal{X}))=\left(V, E_{G}(\mathcal{P})\right)$ is $M$-independent, and hence $\mathcal{X}$ is an independent cover of $G^{2}$. To see this consider a nonempty subset $Z \subseteq V$. If $Z \subseteq P_{i}$ holds for some $1 \leq i \leq t$ then $i_{K}(Z)=0$. Now suppose that $Z$ intersects at least two members of $\mathcal{P}$ and let $\mathcal{Q}=\left\{P_{i} \in \mathcal{P}\right.$ : $\left.Z \cap P_{i} \neq \emptyset\right\}$. By Lemma 3.2(a) we have

$$
5 i_{K}(Z) \leq 5 e_{G}(\mathcal{Q}) \leq 6(|\mathcal{Q}|-1) \leq 6(|Z|-1) .
$$

Hence $i_{K}(Z) \leq \frac{6}{5}(|Z|-1)$ for all nonempty $Z \subseteq V$. Thus $K$ is sparse: every subgraph of $K$ has average degree less than three, and hence has a vertex of degree at most two. This implies that $K$ can be obtained from a collection of disjoint edges (which is $M$-independent) by a sequence of 0 -extensions. Thus $K$ is $M$-independent by Lemma 3.3.

The connection between $\operatorname{val}(\mathcal{X})$ and $\operatorname{def}(G)$ is obtained as follows.

$$
\operatorname{val}(\mathcal{X})=\sum_{i=1}^{t} f\left(X_{i}\right)-\sum_{u v \in H(\mathcal{X})}(h(u v)-1)
$$




$$
\begin{aligned}
& =\sum_{i=1}^{t}\left(3\left|X_{i}\right|-6\right)-\left|E_{G}(\mathcal{P})\right|=\sum_{i=1}^{t} 3\left(\left|P_{i}\right|+d_{G}\left(P_{i}\right)\right)-\left|E_{G}(\mathcal{P})\right|-6 t \\
& =3|V|+6\left|E_{G}(\mathcal{P})\right|-\left|E_{G}(\mathcal{P})\right|-6 t=3|V|+5 e_{G}(\mathcal{P})-6 t \\
& =3|V|-6-\operatorname{def}(\mathcal{P})=3|V|-6-\operatorname{def}(G),
\end{aligned}
$$

as required.

\section{Rank of molecular graphs}

We first show that the right hand side of equation (2) in Conjecture 2.2 gives an upper bound on $r\left(G^{2}\right)$.

Theorem 4.1 Let $G=(V, E)$ be a graph of minimum degree at least two. Then

$$
r\left(G^{2}\right) \leq 3|V|-6-\operatorname{def}(G) .
$$

Proof: Consider a tight partition $\mathcal{P}=\left\{P_{1}, P_{2}, \ldots, P_{t}\right\}$ of $V$. Let $X_{i}=$ $P_{i} \cup N_{G}\left(P_{i}\right)$ for $1 \leq i \leq t$ and let $\mathcal{X}=\left\{X_{1}, \ldots, X_{t}\right\}$. We can use Theorem 3.4 and Lemma 3.1 to deduce that $\mathcal{X}$ is an independent 2-thin cover of $G^{2}$ and $r\left(G^{2}\right) \leq \operatorname{val}(\mathcal{X})=3|V|-6-\operatorname{def}(\mathcal{P})=3|V|-6-\operatorname{def}(G)$, as required.

We next show that Conjecture 2.2 could be used to determine $r\left(G^{2}\right)$, for all graphs $G$, not just graphs of minimum degree at least two. Let $G=(V, E)$ be a connected graph on at least two vertices and let $V_{1}(H)$ denote the set of vertices of degree one in $G$. Let $G_{\text {core }}$ be the maximal subgraph of $G$ of minimum degree at least two. Note that $G_{\text {core }}$ is empty if and only if $G$ is a tree, and $G=G_{\text {core }}$ if and only if $V_{1}(G)$ is empty. Part (a) of the next lemma is due to Franzblau [2].

Lemma 4.2 Let $G=(V, E)$ be a connected graph on at least two vertices.

(a) If $G$ is a tree then $r\left(G^{2}\right)=2|V|-5+\left|V_{1}(G)\right|$.

(b) If $G$ is not a tree then

$$
r\left(G^{2}\right)=r\left(\left(G_{\text {core }}\right)^{2}\right)+2\left|V\left(G-G_{\text {core }}\right)\right|+\left|V_{1}(G)\right| .
$$

Proof: Induction on $|V|$. The theorem is trivially true if $|V|=2$ or $V_{1}(G)=\emptyset$, so we may assume that $V_{1}(G) \neq \emptyset$ and $|V| \geq 3$. Let $v \in V_{1}(G)$, let $H=G-v$, and let $u$ be the neighbour of $v$ in $G$. If $d_{G}(u) \geq 3$, then 
$r\left(G^{2}\right)=r\left(H^{2}\right)+3$ (by Lemma 3.3), $G_{\text {core }}=H_{\text {core }}$ and $\left|V_{1}(G)\right|=\left|V_{1}(H)\right|+1$. On the other hand, if $d_{G}(u)=2$ then $r\left(G^{2}\right)=r\left(H^{2}\right)+2$ (by Lemma 3.3), $G_{\text {core }}=H_{\text {core }}$ and $\left|V_{1}(G)\right|=\left|V_{1}(H)\right|$. In both cases, the lemma follows by applying induction to $H$.

We close this section by showing that Conjecture 2.2 is equivalent to the Molecular Conjecture.

Theorem 4.3 Conjectures 2.1 and 2.2 are equivalent.

Proof: The fact that Conjecture 2.2 implies Conjecture 2.1 follows immediately from Theorem 2.3, as we noted earlier.

Suppose Conjecture 2.1 holds and let $G=(V, E)$ be a graph of minimum degree at least two. We show that Conjecture 2.2 holds for $G$ by induction on $\operatorname{def}(G)$. If $\operatorname{def}(G)=0$, then Theorem 2.3 implies that $5 G$ has six edgedisjoint spanning trees. Since Conjecture 2.1 holds, $G^{2}$ is rigid and hence $r\left(G^{2}\right)=3|V|-6$. Thus Conjecture 2.2 holds for $G$. Hence we may assume that $\operatorname{def}(G) \geq 1$. Let $\mathcal{B}$ be the brick partition of $G$. Since $\operatorname{def}(G) \geq 1$, we have $|\mathcal{B}| \geq 2$. Choose two vertices $u, u^{\prime}$ belonging to distinct bricks $B, B^{\prime} \in \mathcal{B}$, respectively.

Let $G_{1}$ be the graph obtained from $G$ by attaching an ear $u x_{1} x_{2} x_{3} x_{4} u^{\prime}$ of length five at $u, u^{\prime}$, i.e. a path of length five with $x_{1}, x_{2}, x_{3}, x_{4} \notin V(G)$.

Claim $4.4 \operatorname{def}\left(G_{1}\right)=\operatorname{def}(G)-1$.

Proof: Consider the brick partition $\mathcal{B}_{1}$ of $G_{1}$. Since each non-singleton brick has at least three vertices and minimum degree at least two, the vertices $x_{1}, x_{2}, x_{3}, x_{4}$ either each occur as singleton bricks of $G_{1}$, or are all contained in the same brick of $G_{1}$. Since the bricks of $G$ are maximal strong subgraphs of $G$, it follows that $\mathcal{B}_{1}=\mathcal{P}$ or $\mathcal{B}_{1}=\mathcal{P}^{\prime}$, where

$$
\mathcal{P}=\mathcal{B} \cup\left\{\left\{x_{1}\right\},\left\{x_{2}\right\},\left\{x_{3}\right\},\left\{x_{4}\right\}\right\},
$$

and

$$
\mathcal{P}^{\prime}=(\mathcal{B}-\mathcal{Q}) \cup\left\{\left(\bigcup_{B_{i} \in \mathcal{Q}} B_{i}\right) \cup\left\{x_{1}, x_{2}, x_{3}, x_{4}\right\}\right\},
$$

for some $\mathcal{Q} \subseteq \mathcal{B}$ with $B, B^{\prime} \in \mathcal{Q}$. We have

$$
\begin{aligned}
\operatorname{def}_{G_{1}}(\mathcal{P}) & =6(|\mathcal{P}|-1)-5 e_{G_{1}}(\mathcal{P})=6(|\mathcal{B}|+4-1)-5\left(e_{G} \mathcal{B}+5\right) \\
& =\operatorname{def}_{G}(\mathcal{B})-1=\operatorname{def}(G)-1
\end{aligned}
$$


On the other hand, if we let $\mathcal{R}=(\mathcal{B}-\mathcal{Q}) \cup\left\{\bigcup_{B_{i} \in \mathcal{Q}} B_{i}\right\}$ then $\mathcal{R}$ partitions $V,|\mathcal{R}|<|\mathcal{B}|$ since $|\mathcal{Q}| \geq 2$, and

$$
\operatorname{def}_{G_{1}}\left(\mathcal{P}^{\prime}\right)=\operatorname{def}_{G}(\mathcal{R})<\operatorname{def}_{G}(\mathcal{B})=\operatorname{def}(G),
$$

by Lemma 3.2(c). Thus $\operatorname{def}_{G_{1}}\left(\mathcal{B}_{1}\right)=\operatorname{def}(G)-1$. The claim now follows since $\operatorname{def}\left(G_{1}\right)=\operatorname{def}_{G_{1}}\left(\mathcal{B}_{1}\right)$.

It follows from Claim 4.4 that we may apply induction to $G_{1}$ and deduce that

$$
\begin{aligned}
r\left(G_{1}^{2}\right) & =3\left|V\left(G_{1}\right)\right|-6-\operatorname{def}\left(G_{1}\right)=3(|V|+4)-6-(\operatorname{def}(G)-1) \\
& =3|V|-6-\operatorname{def}(G)+13 .
\end{aligned}
$$

Consider the graph $H$ obtained from $G$ by adding the vertices $x_{1}, x_{4}$ and edges $u x_{1}, u^{\prime} x_{4}$. Since the neighbour sets of $u$ and $u^{\prime}$ in $H^{2}$ each induce complete (and hence rigid) subgraphs with at least three vertices, we may use Lemma 3.3 to deduce that $r\left(H^{2}\right)=r\left(G^{2}\right)+6$. This gives

$$
r\left(G_{1}^{2}\right) \leq r\left(H^{2}\right)+\left|E\left(G_{1}^{2}\right)-E\left(H^{2}\right)\right|=\left(r\left(G^{2}\right)+6\right)+7=r\left(G^{2}\right)+13 .
$$

Combining (3) and (4), we obtain $3|V|-6-\operatorname{def}(G)+13=r\left(G_{1}^{2}\right) \leq r\left(G^{2}\right)+13$. Hence $r\left(G^{2}\right) \geq 3|V|-6-\operatorname{def}(G)$. Theorem 4.1 now implies that $r\left(G^{2}\right)=$ $3|V|-6-\operatorname{def}(G)$. Hence Conjecture 2.2 holds for $G$.

\section{$5 \quad$ Independence of molecular graphs}

Recall that a graph $G=(V, E)$ is $M$-independent if $r(G)=|E|$. We say that $G$ is Laman if for all $X \subseteq V$ with $|X| \geq 3$, we have $i_{G}(X) \leq 3|X|-6$. If $G$ is $M$-independent then, since $r(G[X]) \leq 3|X|-6$ for all $X \subseteq V$ with $|X| \geq 3$ by (1), it follows that $G$ is Laman. Jacobs [12] conjectures that the reverse implication also holds for squares of graphs. ${ }^{1}$

Conjecture 5.1 Let $G$ be a graph. Then $G^{2}$ is $M$-independent if and only if $G^{2}$ is Laman.

We will show that Conjecture 5.1 would follow from Conjecture 2.2. We use the following two results.

\footnotetext{
${ }^{1}$ He states this conjecture as a result, [12, Proposition 4.9], but his proof is incomplete since it assumes the truth of [12, Observation 3.1] for which no proof is yet known.
} 
Lemma 5.2 Let $G=(V, E)$ be a graph such that $G^{2}$ is Laman. Then each vertex of $G$ has degree at most three.

Proof: Choose $v \in V$. Then $G^{2}\left[N_{G}(v) \cup\{v\}\right]$ is a complete graph on $d_{G}(v)+1$ vertices. Since the complete graph $K_{n}$ is Laman only when $n \leq 4$, we have $d_{G}(v) \leq 3$.

Theorem 5.3 Suppose that $G$ has minimum degree at least two and $G^{2}$ is Laman. Then $\left|E\left(G^{2}\right)\right| \leq 3|V(G)|-6-\operatorname{def}(G)$.

Proof: We may assume that $\operatorname{def}(G) \geq 1$, since the theorem trivially holds for Laman graphs with deficiency zero. Let $\mathcal{P}=\left\{P_{1}, P_{2}, \ldots, P_{t}\right\}$ be a tight partition of $V$. Since $\operatorname{def}(G) \geq 1$, we must have $t \geq 2$.

An edge $u w \in E\left(G^{2}\right)$ is called a cross edge, if $u w \in E_{G^{2}}(\mathcal{P})$ and $u w \notin$ $E(G)$. Lemma 3.2(a) implies that for every cross edge $u w$ there is a unique pair $u v, v w$ of adjacent edges of $G$ which 'implies' $u w$. Since $u, v$ belong to distinct members of $\mathcal{P}$, at least one of the edges $u v, v w$ must belong to $E_{G}(\mathcal{P})$. We say that the cross edge $u w$ is normal (special) if precisely one (respectively, both) of the edges $u v, v w$ connect distinct members of $\mathcal{P}$. Let $C_{n}$ and $C_{s}$ denote the number of normal and special cross edges of $G^{2}$, respectively. A normal edge $f$ is rooted at $P_{i}$ if one of the two edges of $G$ that 'imply' $f$ is induced by $P_{i}$.

It follows from Lemma 3.2(b) that $G\left[P_{i}\right]$ is strong for all $1 \leq i \leq t$, and hence either $\left|P_{i}\right|=1$, or $\left|P_{i}\right| \geq 3$ and $d_{G\left[P_{i}\right]}(v) \geq 2$ holds for all $v \in P_{i}$. Let $\mathcal{P}_{s}=\left\{P_{i} \in \mathcal{P}:\left|P_{i}\right|=1\right\}$ and let $\mathcal{P}_{b}=\left\{P_{i} \in \mathcal{P}:\left|P_{i}\right| \geq 3\right\}$.

Consider a set $P_{i} \in \mathcal{P}_{b}$ and a cross edge $u v \in E_{G^{2}}(\mathcal{P})$ with $v \in P_{i}$. Since $G^{2}$ is Laman, $d_{G}(v) \leq 3$ by Lemma 5.2 . Thus, since $d_{G\left[P_{i}\right]}(v) \geq 2$, there are exactly two normal cross edges which are 'implied' by pairs $u v, v w$, for some $w \in P_{i}$. Hence the number of normal cross edges rooted at $P_{i}$ is $2 d_{G}\left(P_{i}\right)$. It also follows that if a pair of edges 'implies' a special cross edge then their common vertex cannot belong to $P_{i}$.

Now consider a set $P_{j} \in \mathcal{P}_{s}$. By Lemma $3.2(\mathrm{a}), G^{2}\left[N_{G}\left(P_{j}\right)\right]$ is a complete subgraph consisting of special cross edges, and all special cross edges can be obtained in this way from a unique member of $\mathcal{P}_{s}$. Since $d_{G}(v) \in\{2,3\}$ for all $v \in V$, we have

$$
\left|E\left(G^{2}\left[N_{G}\left(P_{j}\right)\right]\right)\right|=2 d_{G}\left(P_{j}\right)-3 .
$$

By using these observations we can count the normal edges at their roots and special edges in the neighbourhoods of the singleton members of $\mathcal{P}$. This 
gives:

$$
\begin{gathered}
C_{n}=\sum_{P_{i} \in \mathcal{P}_{b}} 2 d_{G}\left(P_{i}\right), \\
C_{s}=\sum_{P_{j} \in \mathcal{P}_{s}}\left|E\left(G^{2}\left[N_{G}\left(P_{j}\right)\right]\right)\right| .
\end{gathered}
$$

Using (5), (6), (7), and the fact that $G^{2}$ is Laman we obtain:

$$
\begin{aligned}
\left|E\left(G^{2}\right)\right| & =\sum_{i=1}^{t}\left|E\left(G^{2}\left[P_{i}\right]\right)\right|+e_{G}(\mathcal{P})+C_{n}+C_{s} \\
& \leq \sum_{P_{i} \in \mathcal{P}_{b}}\left(3\left|P_{i}\right|-6\right)+e_{G}(\mathcal{P})+\sum_{P_{i} \in \mathcal{P}_{b}} 2 d_{G}\left(P_{i}\right)+\sum_{P_{j} \in \mathcal{P}_{s}}\left|E\left(G^{2}\left[N_{G}\left(P_{j}\right)\right]\right)\right| \\
& =\sum_{i=1}^{t}\left(3\left|P_{i}\right|-6\right)+e_{G}(\mathcal{P})+\sum_{i=1}^{t} 2 d_{G}\left(P_{i}\right) \\
& =3|V|-6 t+5 e_{G}(\mathcal{P})=3|V|-6-\operatorname{def}(\mathcal{P})=3|V|-6-\operatorname{def}(G),
\end{aligned}
$$

as claimed.

We can now show that Conjecture 5.1 would follow from Conjecture 2.2.

Theorem 5.4 Conjecture 2.2 implies Conjecture 5.1.

Proof: Suppose, for a contradiction, that $G^{2}$ is Laman but $r\left(G^{2}\right)<\left|E\left(G^{2}\right)\right|$. Since for graphs $G$ of maximum degree at most three $G^{2}$ is $M$-independent if and only if $\left(G_{\text {core }}\right)^{2}$ is $M$-independent, we may assume that $G=G_{\text {core }}$, i.e. $G$ has minimum degree at least two. By using Theorem 5.3 and assuming that Conjecture 2.2 holds for $G$, this gives $\left|E\left(G^{2}\right)\right| \leq 3|V|-6-\operatorname{def}(G)=$ $r\left(G^{2}\right)<\left|E\left(G^{2}\right)\right|$, a contradiction.

Since it is not true that the rigidity of $G^{2}$ implies the existence of a spanning subgraph $H$ of $G$ with the property that $H^{2}$ is both rigid and $M$-independent (consider for example the case when $G$ is a cycle of length five), it seems unlikely that Conjectures 2.1 and 2.2 could be deduced from Conjecture 5.1.

\section{Concluding remarks}

In this paper we have established an upper bound on the rank of a molecular graph and showed that the truth of the Molecular Conjecture would imply 
that this bound is tight. In the companion paper [8] we show that two other conjectures in combinatorial rigidity (due to Dress and Jacobs, respectively) imply the Molecular Conjecture.

Further results on Laman squares as well as sufficient conditions for the $M$-independence of a molecular graph can be found in [7]. In two recent papers $[11,9]$ we obtain results which independently imply a weaker form of the Molecular Conjecture: if $G$ is a graph and $2 G$ has three edge-disjoint spanning trees, then $G^{2}$ is rigid.

\section{References}

[1] D. Franzblau, Combinatorial algorithm for a lower bound on frame rigidity, SIAM J. Disc. Math. Vol. 8, No. 3, pp. 388-400, 1995.

[2] D. Franzblau, Generic rigidity of molecular graphs via ear decomposition, Discrete Applied Math. 101 (2000) 131-155.

[3] J. Graver, B. Servatius, and H. Servatius, Combinatorial Rigidity, AMS Graduate Studies in Mathematics Vol. 2, 1993.

[4] B. JACKSON AND T. JoRDÁN, The $d$-dimensional rigidity matroid of sparse graphs, J. Combin. Theory Ser. B 95 (2005), no. 1, 118-133.

[5] B. Jackson and T. Jordán, The Dress conjectures on rank in the 3dimensional rigidity matroid, Adv. in Appl. Math. 35 (2005), no. 4, 355-367.

[6] B. JACKSON AND T. JORDÁN, On the rank function of the 3-dimensional rigidity matroid, Int. J. Comput. Geom. Appl., Vol. 16, Nos. 5-6 (2006) 415429.

[7] B. JACKSON AND T. JoRDÁN, Rank and independence in the rigidity matroid of molecular graphs, EGRES TR-2006-02, www.cs.elte.hu/egres/

[8] B. JaCkson And T. Jordán, Rigid components in molecular graphs, Algorithmica, 48 (2007) 399-412.

[9] B. JaCKSON AND T. JoRdÁn, Pin-collinear body-and-pin frameworks and the molecular conjecture, submitted. See also EGRES TR-2006-06, www.cs.elte.hu/egres/

[10] B. Jackson And T. Jordán, Brick partitions of graphs, submitted. See also EGRES TR-2007-05, www.cs.elte.hu/egres/

[11] B. JaCKSON AND T. JoRdÁn, The generic rank of body-bar-and-hinge frameworks, submitted. See also EGRES TR-2007-06, www.cs.elte.hu/egres/

[12] D. JACOBS, Generic rigidity in three-dimensional bond-bending networks, $J$. Phys. A, 31, 6653-6668 (1998). 
[13] C.St.J.A. Nash-Williams, Edge-disjoint spanning trees of finite graphs, The Journal of the London Mathematical Society 36 (1961) 445-450.

[14] T.S. TAY, Linking $(n-2)$-dimensional panels in $n$-space II: $(n-2,2)$ frameworks and body and hinge structures, Graphs and Combinatorics 5, 1989, pp. 245-273.

[15] T.S. TAY AND W. Whiteley, Recent advances in the generic rigidity of structures, Structural Topology 9, 1984, pp. 31-38.

[16] W.T. Tutre, On the problem of decomposing a graph into $n$ connected factors, The Journal of the London Mathematical Society 36 (1961) 221-230.

[17] W. Whiteley, The union of matroids and the rigidity of frameworks, SIAM J. Disc. Math. Vol. 1, No. 2, May 1988, pp. 237-255.

[18] W. Whiteley, Some matroids from discrete applied geometry, in Matroid theory (J.E. Bonin, J.G. Oxley and B. Servatius eds., Seattle, WA, 1995), Contemp. Math., 197, Amer. Math. Soc., Providence, RI, 1996, 171-311.

[19] W. Whiteley, Rigidity of molecular structures: geometric and generic analysis, in: Rigidity theory and applications (Edited by Thorpe and Duxbury), Kluwer 1999, pp. 21-46.

[20] W. Whiteley, Rigidity and scene analysis, in: Handbook of Discrete and Computational Geometry (J. E. Goodman and J. O'Rourke, eds.), CRC Press, Second Edition, pp. 1327-1354, 2004.

[21] W. Whiteley, The equivalence of molecular rigidity models as geometric and generic graphs, manuscript, 2004.

[22] W. Whiteley, Counting out to the flexibility of molecules, Physical Biology 2 (2005) S116-S126. 